\title{
CUIDADOS AL ADULTO POSTOPERADO EN LA ADMINISTRACIÓN DE INSULINA
}

CARE FOR THE POSTOPERATIVE ADULT IN THE ADMINISTRATION OF INSULIN.

Recibido 18 de julio 2017 Aceptado 15 de septiembre 2017

Correspondencia: Itzel Leonardo Cruz

Calle Gonzalo Aragón \# 4 Colonia San Lorenzo Huipulco, Tlalpan

Ciudad de México, México Teléfono +52 5557743484 . Correo electrónico: leonardocruzitzel@hotmail.com itzelleonardo2017@gmail.com
Palabras clave: atención de enfermería, administración y dosificación, insulina regular humana, insulina isófana humana, diabetes mellitus tipo 2 , cirugía torácica.

Keywords: Nursing Care, administration \& dosage, Insulin Regular Human, Isophane Insulin Human, Diabetes Mellitus Type 2, Thoracic Surgery.

\section{Autores:}

Itzel Leonardo Cruz

Pasante en Servicio Social de la Licenciatura en Enfermería. Departamento de Investigación en Enfermería del Instituto Nacional de Cardiología Ignacio Chávez. Ciudad de México, México.

Julio César Cadena Estrada

Maestría en Enfermería. Jefe del Departamento de Investigación en Enfermería del Instituto Nacional de Cardiología Ignacio Chávez. Ciudad de México, México 


\section{RESUMEN}

Introducción: Los pacientes adultos postoperados de cirugía cardiaca (POCC), con o sin diabetes mellitus tipo 2 (DM T2), comúnmente presentan descontrol glucémico, favoreciendo la presencia de complicaciones que aumentan la morbimortalidad.

Objetivo: Describir cuidados de enfermería al paciente adulto POCC con o sin DM T2 en la administración de insulina.

Metodología: Revisión sistematizada con metodología integradora. Pasos de la enfermería basada en la evidencia: pregunta clínica; descriptores en ciencias de la salud; atención de enfermería, administración y dosificación, insulina regular humana e insulina isófana humana en español, inglés y portugués; búsqueda de la evidencia científica (SciELO, CINAHL, PubMed, LILACS, CUIDEN), artículos en texto completo publicados del 1 enero 2012 a 31 marzo 2017; lectura y evaluación de la evidencia científica con tabla de evidencia; y análisis de contenido a profundidad para integración de la evidencia

Resultados: se encontraron 20 artículos que cumplieron con los criterios de inclusión. Prevalecieron los estudios con nivel de evidencia observacionales III/C, publicados en países anglosajones en inglés del área médica. Se identificaron cinco dimensiones: valoración de enfermería, medición de la glucosa en sangre, control glucémico, las complicaciones por hipoglucemia o hiperglucemia, y recomendaciones o seguimientos para enfermería.

Conclusiones: los cuidados de enfermería basados en la evidencia, pueden mejorar el control metabólico y prevenir las complicaciones por hipoglucemia e hiperglucemia.

Palabras clave: Atención de enfermería, administración y dosificación, insulina regular humana, insulina isófana humana, diabetes mellitus tipo 2, cirugía torácica.
ABSTRACT

Introduction: Postoperative adult patients with cardiac surgery (POCC) with or without type 2 diabetes mellitus (T2DM) commonly present glycemic uncontrol, favoring the presence of complications that increase morbimortality.

Objective: To describe nursing care for adult POCC patients with or without T2DM in insulin administration.

Methodology: Systematized review with integrative methodology. Evidence-based nursing steps: clinical question; descriptors in health sciences: nursing care, administration and dosing, regular human insulin and human isoform insulin; search for scientific evidence (SciELO, CINAHL, PubMed, LILACS, CUIDEN), full text articles published from January 1, 2012 to March 37, 2017 in Spanish, English and Portuguese; reading and evaluation of scientific evidence with evidence table: and in-depth content analysis for evidence integration.

Results: 20 articles were found that met the inclusion criteria. Prevailing studies with level of observational evidence III / C, published in English in Anglo-Saxon countries of the medical area. Five dimensions were identified: nursing assessment, blood glucose measurement, glycemic control, complications due to hypoglycemia or hyperglycemia and recommendations or follow-ups for nursing.

Conclusions: Evidence-based nursing care can improve metabolic control and prevent complications from hypoglycemia and hyperglycemia.

Key words: Nursing Care, administration \& dosage, Insulin Regular Human, Isophane Insulin Human, Diabetes Mellitus Type 2, Thoracic Surgery. 


\section{INTRODUCCIÓN}

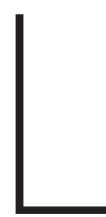

a mayoría de los pacientes que se encuentran hospitalizados en la terapia intensiva postquirúrgica cardíaca (TIQ), pueden presentar alteraciones en los niveles de glucosa sérica llegando a requerir la administración de insulina para el mantenimiento de la glucemia en parámetros normales. En el caso de aquellos pacientes postoperados, de cirugía cardiaca (POCC) con o sin diabetes mellitus tipo 2 (DM T2), quiénes presentan cambios bruscos en los niveles de glucosa en sangre (GS), necesitan de insulina por sistemas de infusión continua (bomba de infusión).

La experiencia clínica muestra que, la mayoría de los pacientes quirúrgicos, pueden mantener un rango de glucemia entre 120 y 180 mg/dL con una infusión de insulina regular que aporte entre 1.0 y 2.0 unidades de insulina por hora. El mecanismo fisiopatológico relacionado al deterioro del control glucémico está asociado, por un lado, a la respuesta metabólica ante el estrés, la técnica anestésica, la cirugía cardiaca, la circulación extracorpórea, el tratamiento farmacológico como glucocorticoides y vasoactivos, que en su conjunto son los factores involucrados en los niveles altos de GS?

Las complicaciones de la hiperglucemia van desde el daño celular y molecular, disfunción endotelial, disfunción del sistema inmunológico, aumento del estrés oxidativo, la apoptosis que junto con la hipoperfusión tisular y pérdida de electrólitos, permiten la aparición de sepsis, alteración en cicatrización, isquemia cerebral, isquemia miocardica, hasta complicaciones renales con retrasos en la recuperación, mayor estancia hospitalaria y aumento en la morbimortalidad. ${ }^{2}$

Por otra parte la administración inadecuada de insulina puede provocar hipoglucemia, desde no grave a grave, causar lesión cerebral irreversible, arritmias cardíacas, convulsiones, coma o la muerte. La magnitud del impacto de hipoglucemia e hiperglucemia en los pacientes en estado crítico y bajo los efectos de sedación, reduce la fiabilidad de los cuidados de enfermería. Por lo anterior, resulta primordial establecer la actuación de enfermería hacia el POCC con o sin DM T2 que se le administra insulina durante su estancia en la unidad de cuidados intensivos ( $\mathrm{UCl}$ ).

Actualmente se han creado algoritmos sobre la terapia de insulina en entornos críticos para la guía y control de hiperglucemia con infusión de insulina, con métodos y efectos diferentes pero no existe un algoritmo, guía o protocolo que permita al profesional de enfermería prevenir resultados adversos asociados con la hiperglucemia en el POCC. Por lo tanto, ante este vacío en el conocimiento, el presente estudió revisión sistematizada con metodología integradora pretendió responder y aportar información a la comunidad de enfermería en relación a los cuidados otorgados a los pacientes adultos POCC con o sin DM T2, en la administración de insulina regular humana y insulina isófana humana (NPH). Con la finalidad de que el paciente reciba los cuidados específicos en la administración de insulina, e integre en su campo de acción, la unificación de criterios de acuerdo a la evidencia disponible para el manejo de la insulina en su control glucémico.

\section{METODOLOGÍA}

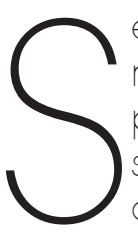
e realizó una revisión sistematizada con la metodología integradora, siguiendo los pasos de la práctica basada en evidencia: selección del tema, pregunta clínica, búsqueda de la evidencia científica, lectura y evaluación de la evidencia científica, integración e interpretación de los resultados. La pregunta clínica generada con la metodología PICOT fue ¿¿cuáles son los cuidados de enfermería otorgados a los pacientes adultos postoperados de cirugía cardiaca con o sin diabetes mellitus tipo 2, en la administración de insulina regular humana y NPH?".

Para la búsqueda de la evidencia, se utilizaron los descriptores en ciencias de la salud (DeCS): "atención de enfermería", "administración y dosificación", "cuidados posoperatorios", "diabetes

80 WWyw Enf Neurol Vol. 16. No. 2 mayo - agosto 2017 
mellitus tipo 2", "cirugía torácica", "insulina", "insulina regular humana" e "insulina isófana humana", en los idiomas español, inglés y portugués; para ampliar la búsqueda se utilizaron los operadores boleanos AND, OR, NOT y, así mismo, se delimitó la búsqueda a todos aquellos artículos en texto completo, publicado del 1 de enero de 2012 al 31 de marzo del 2017, se excluyeron aquellos artículos en otro idioma, tema irrelevante para el estudio, artículos repetidos, artículos sin texto completo.

La búsqueda se realizó en las siguientes bases de datos SciELO, PubMed, CINAHL, CUIDEN y LILACS, el análisis de los datos se realizo a través del contenido a profundidad, utilizando tablas de evidencia y tabla de síntesis que se caracteriza por tener las variables (autor, país, idioma, tipo y diseño de estudio, muestra, intervención, resultados). El nivel de evidencia y grado de recomendación se considero la escala propuesta por la US Agency for Healthcare Research and Quality con modificación del grado de recomendación hecho por SING. ${ }^{3}$

\section{RESULTADOS}

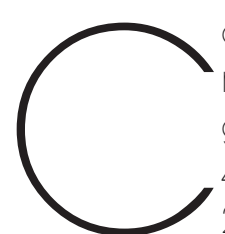

on base en la metodología de la revisión sistematizada con metodología integradora se lograron identificar 4, 032, 937 artículos; no obstante, sólo 20 artículos científicos cumplieron con los criterios de selección mismos que constituyen el cuerpo de este estudio (figura 1).

En relación al área de conocimiento, enfermería sólo generó el 30\%, mientras que medicina lo realizó en el 70\% de los casos. Tomando en cuenta el año de publicación, en el 2015 se publicó la mayoría de artículos con un 30\%, seguido del año 2012 con el 25\% de los artículos que se incluyeron en esta revisión. De acuerdo al diseño y el nivel de evidencia del estudio, prevalecieron los estudios descriptivos/observacionales (III/C) con el $40 \%$ de los casos, seguido de los ensayos clínicos aleatorizados Ib/A con el 20\% y los estudios de revisión sistemática la/A con el 15\% (figura 1).
Por otra parte, la mayoría de los participantes reportados en los artículos encontrados eran adultos POCC 65\%, pero el 15\% corresponde a la participación de enfermeras de la unidad de cuidados intensivos cardiovasculares. Es interesante mencionar que el país que generó más artículos fue Estados Unidos de América 55\% y de Brasil 15\%, pero el resto fue distribuido entre países de Latinoamérica, Europa y África. En relación al idioma, predominó el inglés en el 75\% de los estudios, posteriormente el portugués con 15\%, y el español sólo en un 10\%.

El análisis a profundidad permitió la agrupación de datos en un núcleo temático: el cuidado del POCC con o sin DM T2 que recibe administración de insulina, generando cinco dimensiones: valoración de enfermería, medición de la GS, control glucémico, las complicaciones por hipog/ucemia o hiperg/ucemia, y recomendaciones o seguimientos para enfermería.

En la valoración de enfermería 4 , los factores predisponentes para hiperglucemia son el estrés, injuria aguda por el trauma quirúrgico 5 (efectos de medicamento y técnica anestésica, cirugía cardiaca, circulación extracorpórea), pre existencia de DM T2, descontrol glucémico y/o hemoglobina glucosilada (HbA $1 \mathrm{c}$ ) >8\% después de cirugía, uso de dopamina y adrenalina. ${ }^{1.6}$ Mientras que para la hipoglucemia se encontraron el sexo masculino, edad >65 años, sobrepeso con índice de masa corporal (IMC) $>25-29.9 \mathrm{~kg} / \mathrm{m}^{2}$, hipertensión, cardiopatía isquémica, DM T2, manifestación de insuficiencia renal (IR), hematocrito $<35 \%$, cirugía de emergencia, IR con tratamiento dialítico, la presión parcial de oxígeno en la sangre arterial (PaO2) $\geq 90 \mathrm{~mm} H \mathrm{~g}$ y el empleo de vasoaminas.

En relación a la medición de la GS, la Asociación Americana de Diabetes $(\mathrm{ADA})^{8}$ recomienda mantener niveles entre 140-180 mg/dL de GS en caso de enfermedades críticas y 110-140 mg/ dL en poblaciones seleccionadas con riesgo de hipoglucemia, misma que se define como un nivel de GS <70 mg/dL.? Para medir los niveles de 
GS pueden considerarse las muestras arteriales o venosas según la facilidad de obtención, medirla cada hora en las primeras seis a doce horas del posoperatorio, posterior a esta cada dos horas. Es necesaria la calibración de los dispositivos utilizados para la medición de glucosa como el glucómetro (Accu-Check), este dispositivo tiene una variabilidad del $10 \%$ en tiras reactivas en comparación con los de laboratorio central, la hemogasometría es otra opción, es importante saber que el edema periférico, la hipoperfusión, los fármacos vasoactivos, el hematocrito entre 22\% a 55\% son los factores que pueden influir en la exactitud del resultado de la medición de la GS.,7

Para el control de la GS es recomendable el uso de un protocolo de infusión continua de insulina ${ }^{10}$ en un periodo de 24 a 48 horas," mediante la siguiente escala: si el primer episodio es >150 $\mathrm{mg} / \mathrm{dL}$ pero $<300 \mathrm{mg} / \mathrm{dL}$ se administra un bolo, si es $>300 \mathrm{mg} / \mathrm{dL}$ se inicia infusión; cuando la segunda medición es >150 mg/dL y <250 mg/dL se recomienda tratar con un bolo, pero si es $>250$ mg/dL se inicia una infusión; finalmente, si en una tercera valoración se encuentran niveles > 150 mg/ dL y $<200$ mg/dL se administrará un bolo, pero si los niveles oscilan por arriba de los 200 mg/dL es recomendable iniciar infusión ${ }^{12} \mathrm{o}$, en su caso, se puede hacer el control glucémico por peso, es decir $0.5 \mathrm{UI} / \mathrm{Kg}^{13,14}$

Los predictores para la transición exitosa de insulina intravenosa a subcutánea son variables, pero pueden incluir: cirugía sin complicaciones, pacientes que no requieren ventilación mecánica continua, traslado temprano a la UCl, recepción de 2 unidades/hora o menos de Infusión de insulina tras la conversión a insulina subcutánea, glucemia 130 mg/dL o menos al momento de la transición, y dosis basal de insulina 48 unidades/día o menos, calculado a partir de infusión continua de insulina. ${ }^{15}$ La terapia de insulina intravenosa con insulina regular humana y la insulina NPH intermitente con transición subcutánea fue efectiva, en un estudio permitió alcanzar concentraciones de GS dentro de 70-749 mg/dL. La terapia NPH debe ser implementada con precaución para aquellos que son mayores, y que tienen un control glucémico deficiente. 16

Entre las complicaciones propias de la insulinoterapia, se encuentra la hipoglucemia grave que se caracteriza por niveles de GS menor 40 mg/ dL44 que trae consigo una mayor mortalidad, tratada comúnmente mediante la administración de carbohidratos o glucosa, y la hiperglucemia cuando la GS es mayor o igual a 200 md/dL, que al igual que la hipoglucemia aumentan la morbimortalidad. Cabe mencionar que un control estricto de la GS durante el intraoperatorio trae consigo el riesgo de delirio en el posoperatorio ${ }^{18}$, además la DM T2 se asocia con mayor riesgo de complicaciones cardiacas a los 30 días después de la cirugía cardiaca. Para brindar los cuidados al POCC con o sin DM T2 que recibe insulina se necesita del trabajo en equipo, la experiencia clínica ${ }^{20}$ y la educación continua o capacitaciones que le permitan al profesional de enfermería un mayor empoderamiento para la toma decisiones?

Este estudio permitió el desarrollo de un flujograma con cuidados de enfermería (figura 2), con el objetivo de recomendar un material adicional a la práctica clínica para el cuidado del POCC con o sin DM T2 que recibe la administración de insulina, para el control glucémico de manera segura, permitiendo la estandarización de los cuidados proporcionados y la prevención de complicaciones.

\section{DISCUSIÓN}

I as bases de datos reflejan por excelencia los resultados de los estudios científicos, los avances del conocimiento en las distintas disciplinas y facilitan el intercambio de conocimientos entre investigadores de todos los países. ${ }^{27}$ Por lo tanto, enfermería tiene la oportunidad de aportar a la comunidad científica, vivir el intercambio de conocimientos en el mundo de la investigación.

\section{2} MMN WMy Enf Neurol Vol.16. No. 2 mayo - agosto 2017 
En cuanto al área de conocimiento, enfermería promueve más el desarrollo de estudios descriptivos y observacionales, por lo que se identifica un área de oportunidad en diseñar proyectos de investigación más robustos, con fuerte evidencia para la práctica profesional. Cabe mencionar que la producción científica depende de la población, riqueza y el interés a la investigación, por ejemplo los Estados Unidos de América indica un entorno apto para la investigación científica, Brasil presenta un nivel de producción creciente por su potencial y calidad investigadora, sin embargo, para el resto de los países de América Latina el índice de investigación es menor en la producción científica en enfermería relacionada a esta temática de estudio.

Algunos limitantes para el desarrollo de la investigación en enfermería son la falta de tiempo, falta de formación, el déficit de conocimientos en materia de investigación,22 inclusive factores como la falta de apoyo externo con becas y/o financiamiento, y solo algunos tienen la actitud y aptitud para investigar. ${ }^{23}$ Lo anterior fomentan las barreras, el desinterés al desarrollo de la investigación en los profesionales de enfermería, por lo que existe un bajo número de publicaciones.

Una limitación del presente estudio fue la recuperación de los artículos científicos con texto completo, es triste encontrase con la sorpresa de tener que pagar para tener acceso a una publicación debido a intereses socioeconómicos, lo que hace difícil percibir la labor científica del área de conocimiento en común. Por lo que un área de oportunidad es establecer redes que generen un gran impacto en los organismos para que sea difundido mundialmente, por ejemplo la plataforma researchgate, 24 que busca difundir el conocimiento de una forma más abierta, colaborativa e interactiva.

El idioma inglés goza del primer puesto en términos comparativos con el idioma español y portugués, por ser el idioma oficial para la transmisión y difusión de material en las ciencias de la salud, ${ }^{25}$ por tanto el investigador enfermero debe ser eficiente y superar las fronteras lingüísticas, para asegurar el crecimiento de la producción científica de la enfermería. ¿Por qué quedarse con un solo idioma para la investigación científica?, si el español, el portugués y otros idiomas pueden fortalecer la producción científica y el intercambio de ideas o conocimientos entre investigadores, ampliando el desenvolvimiento y la búsqueda electrónica.

Antes del posoperatorio, una valoración exhaustiva de la historia clínica del paciente permitirá prever las complicaciones postquirúrgicas. Dentro de los hallazgos de este estudio el trauma quirúrgico, el estrés, el tiempo total de anestesia, 5,23 entre otros forman parte de los factores que aumentan la glucosa, causando hiperglucemia, además la HbA 1 C significativamente mayor a $8 \%$ y la diabetes son un predictor del mal control glucémico $y$. junto con el uso de dopamina o adrenalina, 5.6 predisponen las primeras dificultades en el control de la GS. El nivel de GS mayor a 180 mg/dL en las primeros 48 horas, después de la cirugía cardíaca, están asociados con aumentos de infecciones en la herida esternal, además la creatinina alta, la HbA lc alta incrementan el riesgo a corto y largo plazo de morbimortalidad y mayor riesgo de complicaciones en los 30 días del posoperatorio. 16 Sin embargo, la hipertensión, ser diabético, tener hematocrito bajo, incrementa el riesgo 9.6 veces y la cirugía de emergencia la aumenta por siete veces, son variables predisponentes para el desarrollo de la hipoglucemia.,

Debido al control deficiente de la glucosa, la Asociación Americana de Endocrinólogos (AACE) y la ADA sugieren iniciar la infusión de insulina a un umbral de glucemia no superior a $180 \mathrm{mg} / \mathrm{dL}$ y. para el tratamiento de hiperglucemia persistente en pacientes con enfermedad crítica, con un rango objetivo de glucosa en sangre de $140-180$ mg/dL con la finalidad de lograr y mantener un estricto control glucémico en pacientes críticamente enfermos incluidos los de cirugía cardiaca. ${ }^{10}$ El hecho de no llevar a cabo estas recomendaciones aumenta el riesgo de complicaciones para hiperglucemia o hipoglucemia en los pacientes de la UCl.

Enf Neurol Vol. 16. No. 2 mayo - agosto 2017 . 83 
La importancia del control glucémico en el postoperatorio de cirugía cardiaca se centra en evitar complicaciones por hipoglucemia e hiperglucemia, tal y como se encontró en la evidencia, no obstante, en los últimos años el profesional de salud se ha interesado en alcanzar varios objetivos glucémicos para reducir la infección de la herida y las tasas de mortalidad general, ${ }^{15}$ ya que los niveles de glucosa sérica >200 mg/dL en el postoperatorio inmediato (<48 h) contribuyen al aumento del riesgo de infecciones en el sitio quirúrgico. ${ }^{1.4}$

Dentro de las complicaciones encontradas en este estudio, es el desarrollo de delirio posoperatorio por un control estricto de GS en el intraoperatorio, cada aumento de 20 unidades, se asoció significativamente con el inicio de los trastornos neurocognitivos postoperatorios y que las mujeres tenían un riesgo elevado para este resultado. ${ }^{16}$ Además otro estudio muestra la relación del control glucémico con el deterioro cognitivo y la DM T2, así mismo un menor desempeño en la movilidad funcional, la fluidez verbal y una rara com-plicación puede ser el desarrollo de alergia por insulina. ${ }^{27}$

Para prevenir las complicaciones mediante un adecuado control de la GS, se sugiere dar tratamiento a la DM T2 antes de la cirugía cardíaca electiva, por otra parte un estudio recomienda el ayuno con hidratos de carbono antes de la cirugía, ya que mejora el control glucémico en las primeras 6 horas del posoperatorio, con menor estancia hospitalaria, menor uso de dobutamina, y no altera la morbimortalidad hospitalaria. ${ }^{29}$

En enfermería se requieren de líderes que gestionen el cuidado de forma segura y eficaz, con conocimientos innovadores, con la tecnología de vanguardia y el trabajo en equipo para permitir el desenvolvimiento de las habilidades para una atención de calidad; esto es lo esperado, sin embargo, varios estudios informan un déficit de conocimiento con respecto a la glucemia en los médicos asistentes, los residentes médicos y enfermeras de práctica avanzada, por lo que hay la necesidad de educación continua sobre el control de la GS, que permitirá el aumento del conocimiento de las enfermeras de cuidados críticos. 9.17

Una recomendación más es realizar estudios centrados en poblaciones postoperados de cirugía cardiaca con inestabilidad hemodinámica, con presencia de sepsis, o que reciben nutrición enteral o parenteral, terapia con esteroides, por tanto esta población de pacientes puede tener mayor variabilidad de la glucosa, es decir mayor riesgo de hiperglucemia o hipoglucemia.

\section{CONCLUSIONES}

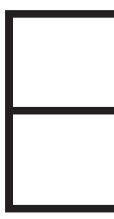

n este estudio se identificaron los cuidados de enfermería como la valoración de los factores de riesgo, la medición y control de la glucosa, la vigilancia y actuación antes las complicaciones por un inadecuado control glucémico; basados en la evidencia científica pueden mejorar el control metabólico de los pacientes POCC con y sin DM T2, así como prevenir o disminuir las complicaciones por hipoglucemia e hiperglucemia.

Este estudio brinda la oportunidad de mejorar la calidad asistencial en los pacientes que reciben insulina, en conjunto con la adaptación de un solo protocolo en hospitales y la reeducación continua del equipo de salud, mejorarán los criterios de tomas de decisiones, permitirá el logro del control glucémico, además de implementar con éxito un cambio en la práctica basado en evidencia científica.

\section{4} LMN/Why Enf Neurol Vol.16. No. 2 mayo - agosto 2017 
Figura 1. Diagrama de búsqueda relacionada con los cuidados de enfermería a pacientes adultos con o sin diabetes mellitus tipos 2, postoperados de cirugía cardiaca en la administración de insulina.

$\mathrm{N}=4,032,937$
Identificados por búsqueda electrónica.

Estudios excluidos
debido a

$n=690,086$ títulos y resúmenes potencialmente relevantes tras el examen inicial.
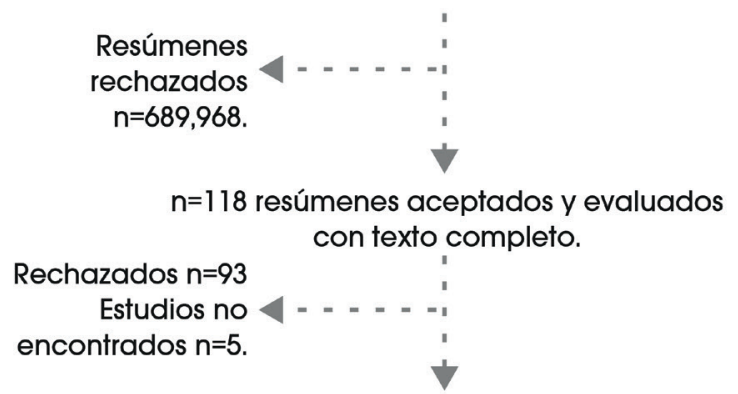

$\mathrm{n}=20$ texto completos $\mathrm{y}$ evaluados.

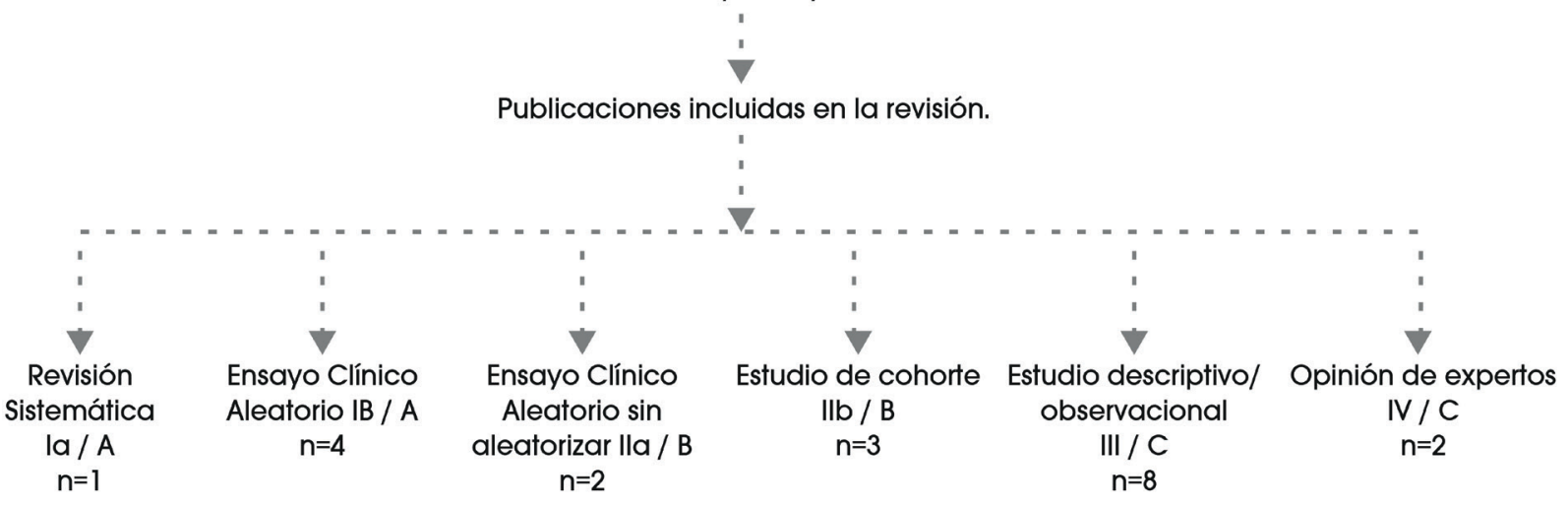



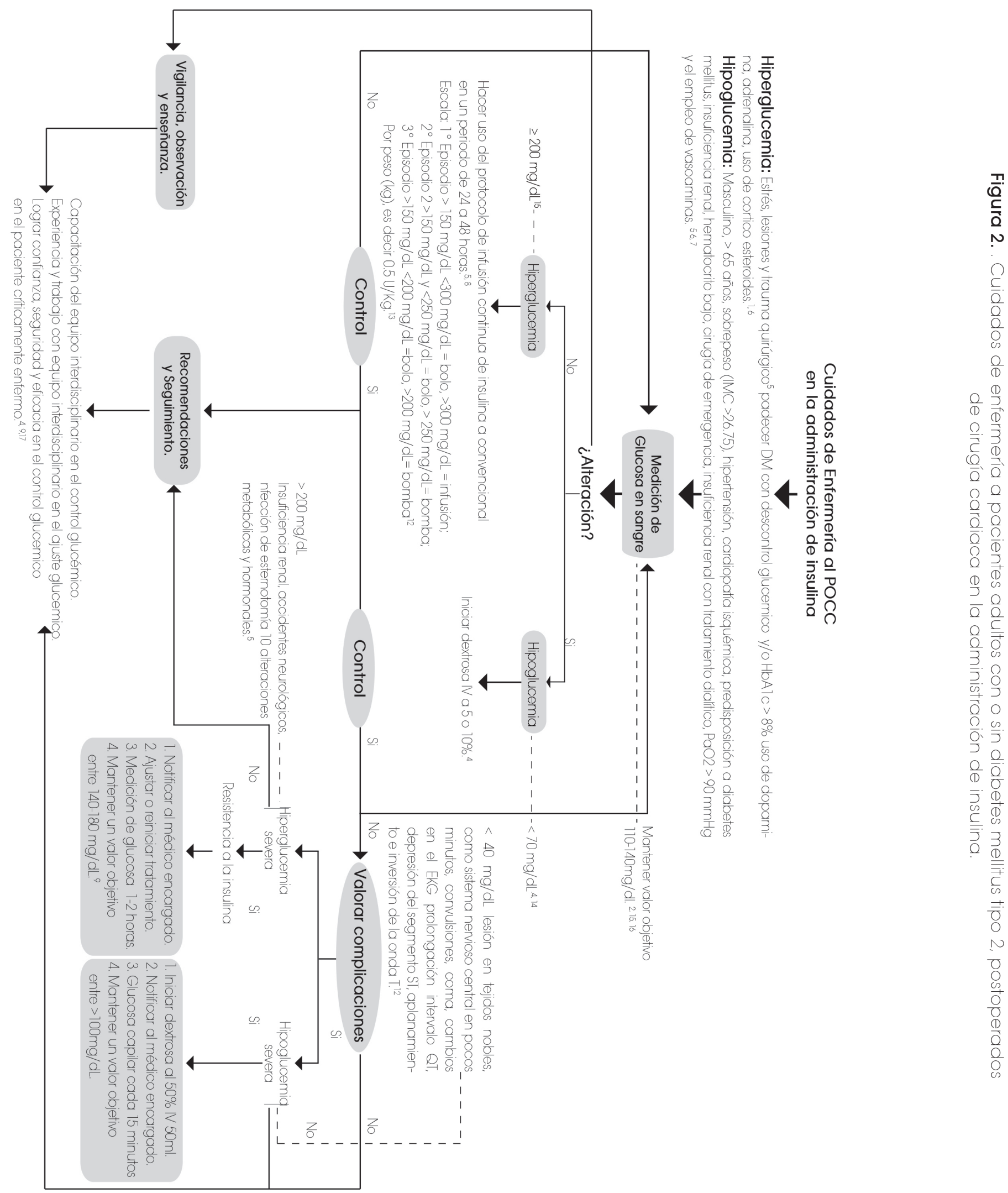

86 MMN/Why Enf Neurol Vol. 16. No. 2 mayo - agosto 2017 
Cuadro 1. Síntesis de la evidencia relacionada con los cuidados de enfermería a pacientes adultos con o sin diabetes mellitus tipos 2, postoperados de cirugía cardiaca en la administración de insulina

\begin{tabular}{|c|c|c|c|c|c|c|c|c|}
\hline Autor & País & Idioma & Metodología & NE/GR & Muestra & Instrumento & Intervención & Resultados \\
\hline Benzadon & Argentina & Español & $\begin{array}{l}\text { Opinión de } \\
\text { expertos }\end{array}$ & $\mathrm{IV} / \mathrm{C}$ & $\begin{array}{c}23 \\
\text { expertos }\end{array}$ & & & $\begin{array}{l}\text { Protocolo dirigido por medico o } \\
\text { enfermera: } 1 \text { episodio }>150 \mathrm{mg} / \mathrm{dL} \\
<300 \mathrm{mg} / \mathrm{dL}=\text { bolo, }>300 \mathrm{mg} / \mathrm{dL} \\
\text { infusión; } 2>150 \mathrm{mg} / \mathrm{dL} \text { y } 250 \\
\mathrm{mg} / \mathrm{dl}=\mathrm{bolo},>250 \mathrm{mg} / \mathrm{dl}=\text { bom- } \\
\mathrm{bq}>150 \mathrm{mg} / \mathrm{dL}<200 \mathrm{mg} / \mathrm{dL}=\text { bolo, } \\
>200 \mathrm{mg} / \mathrm{dL} \text { bomba. }\end{array}$ \\
\hline Rodulfo & Cuba & Español & $\begin{array}{l}\text { Opinión de } \\
\text { expertos }\end{array}$ & $\mathrm{IV} / \mathrm{C}$ & $\begin{array}{c}3 \\
\text { expertos }\end{array}$ & & & $\begin{array}{l}\text { Control metabólico del POCC, } \\
\text { mediante un algoritmo de } \\
\text { diagnóstico. }\end{array}$ \\
\hline Feguri & Brasil & Portugués & $\begin{array}{l}\text { Ensayo Clínico } \\
\text { Aleatorizado }\end{array}$ & $\mathrm{lb} / \mathrm{A}$ & $\begin{array}{c}40 \\
\text { POCC }\end{array}$ & & $\begin{array}{l}\mathrm{Gl}=400 \mathrm{ml} \text { ( } 6 \text { horas } \\
\text { antes) y } 200 \mathrm{ml}(2 \text { horas } \\
\text { antes) de } 12.5 \% \text { de } \\
\text { maltodextrina } \mathrm{G} 2: 400 \\
\text { ml (6 horas antes) y } 200 \\
\text { ml (2 horas antes) } \\
\text { agua sola. }\end{array}$ & $\begin{array}{l}\text { El ayuno con hidratos de carbono } \\
\text { antes de la cirugía, mejora el } \\
\text { control glucemico en las primeras } \\
6 \text { horas del posoperatorio, } \\
\text { < estancia hospitalaria, } \\
\text { < uso de dobutamina, no altera } \\
\text { morbilidad y mortalidad } \\
\text { hospitalaria. }\end{array}$ \\
\hline Nepomuceno & Brasil & Portugués & $\begin{array}{l}\text { Estudio transversal, } \\
\text { prospectivo. }\end{array}$ & $111 / \mathrm{C}$ & $\begin{array}{c}42 \\
\text { POCC }\end{array}$ & & $\begin{array}{l}\text { Insulina intravenosa } \\
100 \mathrm{Ul} / \mathrm{ml}>12 \mathrm{hrs} \mathrm{o}> \\
18 \mathrm{hrs} .\end{array}$ & $\begin{array}{l}\text { Hipoglucemia por hematocrito } \\
\text { bajo, cirugía de emergencia, IRC } \\
\text { con tratamiento dialítico, PaO2 } \geq \\
90 \mathrm{mmHg} \text { y el empleo de } \\
\text { vasoaminas }\end{array}$ \\
\hline Pasión & Brasil & Portugués & $\begin{array}{l}\text { Documental } \\
\text { Retrospectivo }\end{array}$ & $\mathrm{III/C}$ & $\begin{array}{c}550 \\
\text { historias clinicas }\end{array}$ & Encuesta & & $\begin{array}{l}\text { Hipoglucemia severa por ser } \\
\text { masculino, mayor de eddad, } \\
\text { sobrepeso, HAS, Cl, DM T2, IR, } \\
\text { Aminas vasoactivas. }\end{array}$ \\
\hline Chant & USA & Inglés & $\begin{array}{l}\text { Ensayo Clínico } \\
\text { Aleatorizado }\end{array}$ & $\mathrm{Ib} / \mathrm{A}$ & $\begin{array}{c}34 \\
\text { enfermeras }\end{array}$ & & $\begin{array}{l}\text { Juicio de enfermería } \\
\text { (44) vs } \\
\text { Nomograma de } \\
\text { papel (51) }\end{array}$ & $\begin{array}{l}\text { El uso de nomograma de insulina } \\
\text { en papel vs juicio y experiencia de } \\
\text { enfermería, produjo la misma } \\
\text { calidad de control de la glucosa } \\
\text { en los pacientes después de } \\
\text { cirugía cardiaca. }\end{array}$ \\
\hline Westbrook & USA & Inglés & Estudio de Cohorte & $\mathrm{llb} / \mathrm{B}$ & $\begin{array}{l}119 \\
\text { POCC }\end{array}$ & & $\begin{array}{l}\text { Grupo prospectivo: } 60 \\
\text { pacientes con } \\
\text { transición de la insulina } \\
\text { IV en } 24 \text { horas. Grupo } \\
\text { retrospectivo: } 59 \\
\text { pacientes con } \\
\text { transición de la insulina } \\
\text { IV en } 48 \text { horas. }\end{array}$ & $\begin{array}{l}\text { Infusión continúa de insulina IV a } \\
\text { subcutánea en } 24 \text { o } 48 \text { horas igual } \\
\text { de eficaz y seguro. Objetivo } \\
110-150 \mathrm{mg} / \mathrm{dL} \text {. }\end{array}$ \\
\hline Klinker & USA & Inglés & Estudio descriptivo & III/C & $\begin{array}{c}402 \\
\text { POCC }\end{array}$ & & $\begin{array}{l}\text { Pre grupo: } 228 \text { pacientes } \\
\text { control histórico. Pos } \\
\text { grupo: } 174 \text { pacientes } \\
\text { implementación del } \\
\text { protocolo de goteo } \\
\text { de insulina. }\end{array}$ & $\begin{array}{l}\text { El empoderamiento de las } \\
\text { enfermeras y la consulta por parte } \\
\text { de los especialistas en diabetes } \\
\text { puede mejorar la eficiencia en los } \\
\text { procesos de atención y los resultados } \\
\text { clínicos de los pacientes con } \\
\text { cirugía cardíaca. }\end{array}$ \\
\hline Destree & USA & Inglés & Estudio de cohorte & $\mathrm{llb} / \mathrm{B}$ & $\begin{array}{c}161 \\
\text { enfermeras }\end{array}$ & Encuesta & $\begin{array}{l}\text { Pretest : } 2004 \text { Post 1: } \\
\text { 2005, POST2: } 2010\end{array}$ & $\begin{array}{l}\text { La confianza de enfermeras } \\
\text { aumentó con la educación y } \\
\text { la experiencia en curso. }\end{array}$ \\
\hline Nyquist & USA & Inglés & Estudio prospectivo & III/C & $\begin{array}{c}50 \\
\text { POCC }\end{array}$ & & $\begin{array}{l}<70 \mathrm{mg} / \mathrm{dL}, 0>250 \\
\mathrm{mg} / \mathrm{dL} \text { durante } 2 \mathrm{hrs}\end{array}$ & $\begin{array}{l}\text { La valoración de enfermería } \\
\text { permite reconocer los factores } \\
\text { que afectan la glucemia, la } \\
\text { confianza, seguridad y eficacia } \\
\text { en la insulina IV. }\end{array}$ \\
\hline Bakker & Países Bajos & Inglés & Estudio de Cohorte & $\mathrm{llb} / \mathrm{B}$ & $\begin{array}{c:}1462 \\
\text { POOC } \\
239 \\
\text { con DM2 POCC }\end{array}$ & & $\begin{array}{l}\text { Grupo DM T2 con } \\
\text { tratamiento de } \\
\text { insulina: } 87(26 \%) \\
\text { Grupo DM T2 } \\
\text { manejado sin insulina: } \\
242(74 \%) .\end{array}$ & $\begin{array}{l}\text { La DM es una complicación de } \\
\text { mayor riesgo independientemente } \\
\text { de las complicaciones después de } \\
\text { los } 30 \text { días de la cirugía cardiaca. }\end{array}$ \\
\hline
\end{tabular}




\begin{tabular}{|c|c|c|c|c|c|c|c|c|}
\hline Omar & Egipto & Inglés & $\begin{array}{l}\text { Descriptivo } \\
\text { Prospectivo }\end{array}$ & III/C & $\begin{array}{c}227 \\
\mathrm{POCC}\end{array}$ & & $\begin{array}{l}\text { Grupos I (TIR> } 80 \%) \text { : } \\
146 \text { pacientes sin DM } \\
\text { y grupo II (TIR <80\%): } \\
81 \text { pacientes con DM. }\end{array}$ & $\begin{array}{l}\text { La hiperglucemia fue significativamente } \\
\text { más frecuente en los diabéticos } \\
(p=0,001) \text {, en los pacientes con } \\
\text { hemoglobina glicosilada }(\mathrm{HbAlc})> \\
8 \%(P=0,0001) \text {, y en } \\
\text { pacientes que tomaron dopamina } \\
(p=0,04) \text { y adrenalina }(p=0,05) \text {. }\end{array}$ \\
\hline Hargraves & USA & Inglés & $\begin{array}{l}\text { Estudio controlado } \\
\text { sin aleatorizar }\end{array}$ & Ila/B & $\begin{array}{c}29 \\
\text { enfermeras }\end{array}$ & Cuestionario & $\begin{array}{l}\text { Ajuste del protocolo } \\
\text { Yale } 120-160 \mathrm{mg} / \mathrm{dl} \mathrm{a} \\
>150 \mathrm{mg} / \mathrm{dL} .\end{array}$ & $\begin{array}{l}\text { El conocimiento de enfermería de } \\
\text { cuidados críticos en el control de } \\
\text { la glucemia en la enfermedad } \\
\text { crítica aumentó. }\end{array}$ \\
\hline Magaji & USA & Inglés & $\begin{array}{c}\text { Estudio } \\
\text { Retrospectivo }\end{array}$ & III/C & $\begin{array}{c}247 \\
\text { POCC }\end{array}$ & & $\begin{array}{l}\text { Protocolo } 1120-160 \\
\text { mg/dL ( } n=117 \text { pacientes) } \\
\text { eran mayores. } \\
\text { Protocolo } 2110-140 \\
\text { mg/dL. ( } n=130 \\
\text { pacientes). } \\
\text { Cambios de dosis de } \\
\text { insulina para alcanzar } \\
\text { los niveles } \\
\text { objetivos de glucosa } \\
\text { en sangre en pacientes } \\
\text { tras cirugía cardíaca. }\end{array}$ & $\begin{array}{l}\text { El protocolo de infusión continúa } \\
\text { de insulina dirigido a } 110-140 \\
\mathrm{mg} / \mathrm{dL} \text { fueron eficaces para lograr } \\
\text { objetivos revisados con hipoglucemia } \\
\text { baja. }\end{array}$ \\
\hline Borelan & USA & Inglés & $\begin{array}{l}\text { Revisión } \\
\text { sistemática }\end{array}$ & $\mathrm{Ia} / \mathrm{A}$ & $\begin{array}{c}11 \\
\text { artículos }\end{array}$ & $\begin{array}{l}\text { Evaluación y } \\
\text { Revisión } \\
\text { (JBI-MAStARI) }\end{array}$ & & $\begin{array}{l}\text { Reducción de las tasas de infección } \\
\text { del sitio quirúrgico en comparación } \\
\text { con la administración estándar en } \\
\text { pacientes con DM }\end{array}$ \\
\hline Saager & USA & Inglés & $\begin{array}{l}\text { Ensayo Clínico } \\
\text { Aleatorizado }\end{array}$ & $\mathrm{lb} / \mathrm{A}$ & $\begin{array}{c}198 \\
\text { POCC }\end{array}$ & & $\begin{array}{l}\text { Pacientes asignados } \\
\text { aleatoriamente para } \\
\text { el control con hiperinsu- } \\
\text { linémico-normo- } \\
\text { glucémico pacientes } \\
\text { (glucosa en sangre } \\
\text { objetivo: } 80-110 \mathrm{mg} / \mathrm{dL} \\
\text { (n=93)) o } \\
\text { (Administración } \\
\text { convencional de } \\
\text { insulina con un objetivo } \\
\text { de glucosa en sangre } \\
<150 \mathrm{mg} / \mathrm{dL}(\mathrm{n}=105) \text { ). }\end{array}$ & $\begin{array}{l}\text { La intervención hiperinsulinémico } \\
\text { normoglucemia en el intraoperatorio } \\
\text { aumenta el riesgo de delirio } \\
\text { después de la cirugía cardíaca, } \\
\text { pero no su gravedad. }\end{array}$ \\
\hline Mulla & USA & Inglés & $\begin{array}{l}\text { Revisión } \\
\text { retrospectiva }\end{array}$ & III/C & $\begin{array}{r}1325 \\
\text { POCC }\end{array}$ & & $\begin{array}{l}80 \text { a } 110 \mathrm{mg} / \mathrm{dL} \text { de } \\
\text { grupo }(n=667) \\
110 \text { a } 140 \mathrm{mg} / \mathrm{dL} \text { grupo } \\
(n=658)\end{array}$ & $\begin{array}{l}\text { Hipoglucemia significativamente } \\
\text { menor y sin diferencias significativas } \\
\text { en mortalidad / morbilidad, con el } \\
\text { objetivo de } 110-140 \mathrm{mg} / \mathrm{dL} \text {. }\end{array}$ \\
\hline Silinskie & USA & inglés & $\begin{array}{l}\text { Ensayo Clínico } \\
\text { Aleatorizado }\end{array}$ & $\mathrm{lb} / \mathrm{A}$ & $\begin{array}{c}80 \\
\mathrm{POCC}\end{array}$ & & $\begin{array}{l}\text { Transición IV a SC. } \\
\text { Grupo basado en peso } \\
(n=40) \text { : recibieron } 0,5 \\
\text { unidades } / \text { kg por vía } \\
\text { subcutánea. Grupo } \\
\text { basado en porcentaje } \\
(n=40) \text {. }\end{array}$ & $\begin{array}{l}\text { La dosificación de insulina glargina } \\
\text { en peso demostró ser segura. } \\
\text { Control con } 0.5 \mathrm{U} / \mathrm{kg} \text { infusión } \\
\text { continuta a SC. }\end{array}$ \\
\hline Chawla & India & Inglés & $\begin{array}{c}\text { Estudio } \\
\text { Observacional }\end{array}$ & III/C & $\begin{array}{c}203 \\
P O C C\end{array}$ & & $\begin{array}{l}\text { Pacientes recibieron } \\
\text { IAsp IV }(n=103) \text { e RHI } \\
(n=100)\end{array}$ & $\begin{array}{l}\text { Aspartosa IV es eficaz y segura para } \\
\text { el manejo de la hiperglucemia en } \\
\text { pacientes en UCl después de los } \\
\text { procedimientos cardíacos, mayor } \\
\text { ventaja en transición y manejo de IV } \\
\text { a SC. }\end{array}$ \\
\hline Liou & China & Inglés & $\begin{array}{l}\text { Estudio controlado } \\
\text { sin aleatorizar }\end{array}$ & $\| a / B$ & $\begin{array}{c}50 \\
\mathrm{POCC}\end{array}$ & & $\begin{array}{l}\text { El grupo INIT, } n=20 \text {, } \\
\text { recibió infusión de } \\
\text { insulina de Yale y } \\
\text { mantener un nivel de } \\
\text { glucosa en sangre } \\
\text { entre } 90-119 \mathrm{mg} / \mathrm{dL} \text {. } \\
\text { El grupo COIT, n=30 } \\
\text { recibió una escala } \\
\text { convencional de } \\
\text { insulina después de } \\
\text { CABG y mantener un } \\
\text { nivel de glucosa en } \\
\text { sangre entre } 180-200 \\
\text { mg/dL }\end{array}$ & $\begin{array}{l}\text { La insulinoterapia intensiva redujo la } \\
\text { glucosa en la sangre y condujo a } \\
\text { mejorar el gasto cardíaco después } \\
\text { de la CABG en comparación con la } \\
\text { insulinoterapia } \\
\text { convencional. }\end{array}$ \\
\hline
\end{tabular}


Cuadro 2. Síntesis de la evidencia por revista relacionada con los cuidados de enfermería a pacientes adultos con o sin diabetesmellitus tipos 2, postoperados de cirugía cardiaca en la administración de insulina.

\begin{tabular}{|c|c|c|c|c|}
\hline No. & Nombre de la revista & Idioma & País & Área \\
\hline 1 & Medicina & Español & Argentina & Médico \\
\hline 2 & MEDISAN & Español & Cuba & Médico \\
\hline 3 & Rev Bras Cir Cardiovasc & Portugués & Brasil & Médico \\
\hline 4 & Revista Enfermagem UERJ & Portugués & Brasil & Médico \\
\hline 5 & Revista Enfermagem UERJ & Portugués & Brasil & Médico \\
\hline 6 & American Journal of Critical Care & Inglés & USA & Enfermería \\
\hline 7 & Journal Of Nursing Care Quality & Inglés & USA & Enfermería \\
\hline 8 & The Journal For Advanced Nursing Practice & Inglés & USA & Enfermería \\
\hline 9 & AACN Advanced Critical Care & Inglés & USA & Enfermería \\
\hline 10 & Dimensions Of Critical Care Nursing & Inglés & USA & Enfermería \\
\hline 11 & Anaesthesia \& Intensive care & Inglés & Países Bajos & Médico \\
\hline 12 & BMC Anesthesiology & Inglés & Egipto & Médico \\
\hline 13 & American journal of critical care & Inglés & USA & Médico \\
\hline 14 & Diabetes Technology \& Therapeutics & Inglés & USA & Médico \\
\hline 15 & Heart \& Lung & Inglés & USA & Médico \\
\hline 16 & Anesthesiology & Inglés & USA & Médico \\
\hline 17 & Diabetes Care & Inglés & USA & Médico \\
\hline 18 & Annals Of Pharmacotherapy & Inglés & USA & Médico \\
\hline 19 & Journal of Diabetes \& Metabolic Disorders & Inglés & India & Médico \\
\hline 20 & Chinese Journal of Physiology & Inglés & China & Enfermería \\
\hline
\end{tabular}




\section{REFERENCIAS BIBLIOGRÁFICAS}

1. Klinkner G. Murray M. Clinical nurse specialists lead teams to impact Glycemic control after cardiac surgery. Clin Nurse Spec. Jul 2014; 28(4): 240-6

2. Mulla I, Schmidt K, Cashy J, Wallia A, Andrei AC, Oakes J, et al. Comparison of glycemic and surgicaloutcomes after change in glycemic targets in cardiac surgery patients. Diabetes Care. Nov 2014; 37(11): 2960-5.

3. Scottish Intercollegiate Guidelines Network. SIGN 50. A guideline developer's handbook. (consultado en mayo de 2017). Disponible en: nttp://bit.ly/2ptc50s

4. Nyquist SK, Anderson JL, Donahue RH, Caruso E, Alore ML, Larson JS. Nurse-driven titration of continuous insulin infusion in post-cardiac surgery patients. Dimens Crit Care Nurs. May 2012; 31 (3): 188-92.

5. Nepomuceno RM, Silva LD, Pereira SRM. Characterísticas dos pacientes críticos que apresentaram hipoglicemia: uso seguro da infusão continua de insulina. R Enferm UERJ. Mar 2015 23(2): 149-55.

6. Omar AS, Salama A, Allam M, Elgohary Y, Mohammed S, Tuli AK, et al. Asociación de tiempo en el rango de glucosa en sangre con los resultados después cirugía cardiaca. BMC Anesthesiol (Internet). Ene 2015 (citada 31 marzo 2017); 15(1): (aprox. 14 p). Disponible en: http://bit.ly/2tdOJ5S

7. Pasión CT, MRI Nepomuceno, MM Santos, Silva LD. Factores predisponentes para hipoglicemia: aumentando a segurança do paciente crítico que utiliza insulina intravenosa. R Enferm UERJ. Jun 2015; 23(7): 70-5.

8. Magaii V, Nayak S, Donihi A, Willard L, Jampana S, Korytkowski M, et al Comparison of Insulin Infusion Protocols Targeting 110-140 mg/dL in Patients After Cardiac Surgery. Diabetes Technol Ther. Nov 2012; 14(11): 1013-7.

9. Hargraves JD. Glycemic control in cardiac surgery: implementing anevidence-based insulin infusion protocol. Am J Crit Care. May 2014 23(3):250-8.

10. Rodulfo GM, De Dios LJA, Rodríguez AOD, Torrallbas RFE. Algoritmo para el control metabólico del paciente diabético en el periodo posoperatoric de la cirugía cardiovascular. MEDISAN. 2014; 18 (7):1023-6.

11. Westbrook A, Sherry D, McDermott M, Gobber M, Pabst M. Examining IV Insulin Practice Guidelines in the Cardiac Surgery Patient. J Qual Care Nursing. Oct 2016; 31 (4): 344-9

12. Benzadón NM, Cohen AH, Comignani P, Villareal Ri, Trivi M, Fuselli JJ, et al. Control de la glucemia en el postoperatorio de cirugía cardíaca: Informe del Consejo de Emergencias de la Sociedad Argentina de Cardiología. Medicina. 2012 Feb; 72(1): 58-62

13. Silinskie K, Kirshner R, Hite M. Converting continuous insulin infusion to subcutaneous insulin glargine after cardiac surgery using percentage based versus weight-based dosing: a pil ot trial. Annals Of Pharmacotherapy Ene 2013; 47(1): 20-8

14. Chant C, Mustard M, Thorpe K, Friedrich J. Nurse-vs nomogram-directed glucose control in a cardiovascular intensive care unit. Am J Crit Care. Jul 2012: 21 (4): 270-79

15. Liou HL, Shih CC, Chung KC, Chen HI. Comparison of the effect of intensive versus conventional insulinotherapy in patients with cardiac surgery after cardiopulmonary bypass. Chin J Physiol. May 2013; 5(2): 101-9.
16. Chawla M, Malve H, Shah H, Shinde S, Bhoraskar A. Safety of intravenous insulin aspart compared to regular human insulin in patients undergoing CU monitoring post cardiac surgery: an Indian experience. J Diabetes Metab Disord (Internet). Abr 2015 (citado 28 marzo 2017); 14: ( aprox. 5 p). Disponible en: http://bit.ly/2dRVMGh

17. Boreland L, Scott-Hudson M, Hetherington K, Frussinetty A, Slyer J. The effectiveness of tight glvcemic control on decreasing surgical site infections and readmission rates in adult patients with diabetes undergoing cardiac surgery: A systematic review. Heart \& Lung. Sep 2015; 44(5): 430-40

18. Saager L, Duncan A, Yared J, Hesler B, You J, Kurz A, et al. Intraoperative tight glucose control using hyperinsulinemicnormoglycemia increases delirium after cardiac surgery. Anesthesiology.Jun 2015; 122(6): 1214-23

19. Bakker E, Valentijn T, Van De Luijtgaarden K, Hoeks S, Voute M, Stolker R, e al. Type 2 diabetes mellitus, independent of insulin use, is associated with an increased risk of cardiac complications after vascular surgery. Anaesthesia \& Intensive care. Sep 2013 41 (5): 584-90

20. Destree L, Ludwig BP, Vercellino M, Rowe A. Effects of education on nurse confidence and cardiac surgical glycemic control. AACN Advanced Critical Care. Oct 2013; 24(4):362-9.

21. Torregrosa JNE. El artículo científico que debemos escribir y como escribirlo. Verba luris (Internet).Ene 2015 (citada 21 abril 2017):33: 11-14 Disponible en: http://bit.ly/20xEltQ

22. Castro TJ. Producción científica en enfermería de urgencias cardíacas y coronarias: Análisis de la literatura. Enferm. glob. (Internet). Oct 2010 (citado 21 abril de 2017); 20: (Aprox 10 p). Disponible en: http://bit.ly/20czp85

23. Cadena-Estrada JC, Olvera-Arreola SS, Teniza-Noguez D, López FL Factores que influyen en el profesional de enfermería de una institución de tercer nivel para desarrollar investigación. INQUIETUDES. Jul 2014; 48: 28-37.

24. Campos-Freire F, Rúas-Araújo J. Uso de las redes sociales digitales profesionales y científicas: el caso de las 3 universidades gallegas. El profesional de la información. 2015 25(3): $437-40$

25. Larenas SMME. La lengua inglesa y su importancia en la investigación en enfermería. Cienc. Enferm. Abr 2016; 22(1): 7-9.

26. Miranda F, Valles OPE, Ortiz FPM, Rosario E. Relación del Contro Glucémico, Función Cognitiva y las Funciones Ejecutivas en el Adulto Mayor con Diabetes Tipo 2: Revisión Sistemática. Revista Electrónica trimestral de Enfermería. Abr 2016; (42): 472-80

27. Andrade P, Goncalo M. Type 1 lg-E mediated allergy to human insulin insulin analogues and beta-lactam antibiotics. An. Bras. Dermatol. 2012; 87 (6):917-19

28. Lee G, Wyatt S, Topliss D, Walker K, Stoney R. A study of a pre-operative intervention in patients with diabetes undergoing cardiac surgery. Collegian. Dic 2014; 21(4): 287-93

29. Feguri GR, Lima PRL, Lopes AM, Roledo A, Marchese M, Trevisan M, et al. Resultados clínicos e metabólicos da abreviação do jejumcomcarboidratos na revascularização cirúrgica do miocárdio. Rev Bras Cir Cardiovasc. 2012 27(1):7-17
90 MNVMW 